\title{
Bodily Tides
}

\author{
Rosemary A. Mardling ${ }^{1}$ \\ ${ }^{1}$ School of Mathematical Sciences [rosemary.mardling@monash.edu]
}

\begin{abstract}
The equilibrium tide model in the weak friction approximation is used by the binary star and exoplanet communities to study the tidal evolution of short-period systems. However, each uses a slightly different approach which potentially leads to different conclusions about the timescales on which various processes occur. Here we present an overview of these two approaches, and show that for short-period planets the circularization timescales they predict differ by at most a factor of a few. A discussion of the timescales for orbital decay, spin-orbit synchronization and spin-oribt alignment is also presented.
\end{abstract}

\section{Two-body tidal evolution}

The effects on an orbit due to tidal distortion and rotation can be divided into two categories: those associated with the viscosity of the fluid (and rheology in the case of a rigid component), and those associated with the non-spherical shape of the distorted body. The latter give rise to apsidal advance of the orbit in its plane in the case that the orbit is non-circular, and to precession of the orbital plane and the spin axes of the rotating bodies about the direction of the total angular momentum of the system in the case that the spin axes are not aligned with the orbit normal. They do not affect the semimajor axis or the eccentricity of the orbit, and occur on a timescale which is generally much shorter than those due to tidal dissipation. In contrast, viscous dissipation in the tidally distorted bodies results in changes to all the orbital elements (except the longitude of periastron) as well as the rotation rate, and is the focus of the so-called equilibrium tide model.

The equilibrium tide model in the weak friction approximation was devised by Darwin (1879) for the purpose of studying tides in planets and their satellites, and in particular the evolution of the orbital elements due to their resulting non-sphericity and orientation. The shape of the tidal bulge is obtained by assuming hydrostatic equilibrium within the distorted body, that is, the fluid's internal pressure gradients and viscous stresses are exactly balanced by the gravitational force exerted both by the fluid body itself and the companion, implying there is no relative motion of neighbouring fluid elements and hence no viscous heat loss. In fact, as long as the orbit is noncircular (the gravitational field of the companion varies in time), and/or the fluid body rotates at a rate different to the mean motion (fluid elements vary their distance to the companion at a rate equal to the difference between the rotation rate and the mean motion, even if the orbit is circular), and/or the rotation axis points in a different direction to the orbit normal (again the gravitational field varies), the assumption of hydrostatic equilibrium is only approximate. However, one can study the effect of 
the tidal dissipation on the orbital evolution without studying the fluid motion itself, simply by realizing that dissipation within the fluid will cause its response to be out of phase with the tidal forcing, this producing a misalignment between the tidal bulge and the line of centres and consequently a spin-orbit torque. The misalignment angle $\epsilon$ is directly related to the dissipation rate in the fluid via the tidal quality factor or $Q$ value $^{1}$ such that $2 \epsilon=Q^{-1}$, and if one assumes that the height of the tide is unaffected by friction (this is the weak-friction approximation), one can calculate the rates of change of the orbital elements by considering the motion of a companion moving in the potential of the obtained figure of equilibrium, the latter slightly rotated with respect to the line of centres. Note that a nice feature of Darwin's model is that in spite of the presence of friction, one can write down a potential and thus make use of Lagrange's planetary equations for the rates of change of the elements.

Darwin's original treatment (and most subsequent treatments) involves a Fourier expansion of the tidal potential, and it is the treatment of each individual Fourier component which distinguishes the two equilibrium tide models in common use; the so-called constant time-lag model which Darwin himself employed assumes that the lag angle of (and hence the rate of dissipation in) a particular tidal component is proportional to the rate at which it is forced, and that the constant of proportionality (the lag time) is common to all tidal components, while the constant lag angle model (Goldreich \& Soter 1966) assumes that all tidal components lag behind (or ahead of) the line of centres by the same angle. Darwin's prescription has been improved over the years by various authors, and adopted by the binary star community as appropriate for studying tides in stars. In contrast, the planet community has adopted the prescription of Goldreich \& Soter (1966) for solid planets and satellites, one which appeals to the experimental fact that for various Earthly solid materials, the lag angle is independent of the forcing frequency for a wide range of forcing frequencies. Moreover, the binary star community refer to the apsidal motion constant, $k_{A}$, a quantity reflecting the degree of central concentration of a fluid body, while the planet community refer to the Love number, $k_{L}$, a quantity associated with the degree of rigidity and other properties of a homogeneous solid body. In fact, they are both (apart from a factor of two) the ratio of the potential associated with the tidal bulge to the tidal potential produced by the perturber at the surface of the distorted object, and are such that $k_{L}=2 k_{A}$.

The choice of prescription for dissipation in a tidally distorted planet - constant lag time or constant lag angle - turns out to vary the tidal circularization timescale by no more than a factor of a few, at least in the simple equilibrium tide model as we show below. Moreover, contrary to the situation to date, it seems more reasonable to use the Darwin prescription in the case of gas giants and Neptune-like ice giants, given that tidal energy is probably mostly stored and dissipated in the dense atmosphere of the latter. For example, it may be that this factor of a few is all that is needed to explain the significantly non-circular orbit of the short-period hot Neptune GJ436b (Butler et al. 2004).

Perhaps the most succinct and elegant derivation of the equations governing the variation of the orbital elements due to tidal friction in stars is given by Hut (1981) who follows Darwin in assuming a constant time lag and weak friction, but unlike Darwin and most others, uses energy and angular momentum arguments instead of a Fourier decomposition of the tidal field. He shows that, in this approximation, one is

\footnotetext{
${ }^{1}$ See Goldreich $(1963$, p260) for a derivation of the relationship between the lag angle of the $Q$-value, as well as http://online.kitp.ucsb.edu/online/exoplanets10/mardling/ for a comparison of the Darwin and Goldreich analyses including a derivation of the Fourier components of the tidal bulge.
} 
able to write down closed-form expressions for the rates of change of the elements and the rotation frequency. ${ }^{2}$ In contrast, the Goldreich \& Soter (1966) constant lag angle theory is correct to first order only in the eccentricity, and does not display explicit dependence on the spin frequencies. In particular, the sign of the rate of change of eccentricity is decided by the sign of the dominant contributing tidal component, this being the one with forcing frequency $2 \Omega-3 n,{ }^{3}$ where $\Omega$ and $n$ are the spin frequency and the mean motion respectively (Goldreich 1963, p261). As such the relevant forcing frequency for a synchronously rotating body $(\Omega=n)$ in a non-circular orbit is the mean motion itself (since $2 \Omega-3 n=-n$ in this case). ${ }^{4}$ This makes sense since a particle in the tidal bulge, although always lying (approximately) at a constant angle away from the line of centres, nonetheless experiences a gravitational field which varies in strength with a frequency equal to the mean motion as its distance to the companion varies over the orbit.

To first order in the eccentricity, the Hut (1981) expression for the rate of change of eccentricity for a synchronously rotating system is given by

$$
\frac{1}{e} \frac{d e}{d t}=-\frac{21}{4} \frac{k_{L}}{T}\left(\frac{M_{2}}{M_{1}}\right)\left(1+\frac{M_{2}}{M_{1}}\right)\left(\frac{R_{1}}{a}\right)^{8},
$$

while the Goldreich \& Soter (1966) expression is ${ }^{5}$

$$
\frac{1}{e} \frac{d e}{d t}=-\frac{21}{2} \frac{n k_{L}}{Q}\left(\frac{M_{2}}{M_{1}}\right)\left(\frac{R_{1}}{a}\right)^{5},
$$

where $n$ is the mean motion, $R_{1}$ and $M_{1}$ are the radius and mass of the tidally distorted body, $M_{2}$ is the mass of the tide-raising body, $T$ is a damping timescale which is related to the time lag $\tau$ via $T^{-1}=\left(G M_{1} / R_{1}^{3}\right) \tau$, and $Q$ is the tidal quality factor. These two expressions agree to this order in eccentricity if we take $\frac{1}{2} n \tau=Q^{-1}$, consistent with the mean motion being the relevant forcing frequency for a synchronously rotating body. ${ }^{6}$

In order to use the constant time lag model to study the tidal evolution of hot Jupiters, while at the same time use information we have about our own Jupiter, we might assume that, instead of the $Q$-value itself, it is $\tau$ which is common to Jupiter and other gas giants of similar mass, although in reality this quantity can be expected to vary from one system to the next according to the planet's density and chemical composition. Since we expect all hot Jupiters to be essentially synchronously rotating, ${ }^{7}$

\footnotetext{
${ }^{2}$ Note that Hut's expression for the rate of change of spin obliquity is valid for small values of this quantity, while Eggleton, Kiseleva and Hut (1998) derive close-form expressions for the rates of change of all three Euler angles associated with rotation.
}

${ }^{3}$ In a frame rotating at the same rate as the tidally distorted body; recall that what is relevant is the relative motion of neighbouring fluid parcels.

${ }^{4}$ It is interesting to note that the Darwin/Hut expression for the rate of change of the eccentricity is proportional to $11 \Omega-18 n$; compare this to $2 \Omega-3 n$.

${ }^{5}$ Note that Goldreich \& Soter (1966) employ a modified $Q$-value involving the Love number and defined by $Q^{\prime}=\frac{3}{2} Q / k_{L}$.

${ }^{6}$ Note that in order to study the case of synchronous rotation, Goldreich (1963) assumes a constant time lag for two of the tidal components and Goldreich \& Soter (1966) go on to assume a constant lag angle for the remainder, thus employing a curious mixture of both formulations.

${ }^{7}$ In fact, for finite eccentricity they will be rotating slightly sub-synchronously (Hut, 1981, p131). 
we can use the relation $Q^{-1} \propto n \tau$ to estimate the $Q$-value of a planet whose orbital period is $P$ via $Q / Q_{J}=P / P_{I o}$, where $Q_{J}$ is Jupiter's $Q$-value and $P_{I o}=1.77 \mathrm{~d}$ is Io's orbital period. Lainey et al (2009) have estimated a value of $Q / k_{L}=9.1 \times 10^{4}$ for Jupiter by astrometrically measuring Io's acceleration. Thus, for example, a hot Jupiter with the same Love number as Jupiter's but with a period of 4 days will have a $Q$-value which is 2.3 times that for Jupiter. Note, however, that if such a hot Jupiter is inflated its Love number will be reduced (as is also the case if the planet has a substantial core), and this will further increase the circularization timescale.

\section{Timescales}

The minimum-energy state of a binary system is one in which the orbit is circular, and both bodies spin synchronously with the mean motion and their spin axes are aligned with the orbit normal. The semimajor axis may increase or decrease during this process, depending on the net transfer of angular momentum. For example, while spin synchronization of a planet by a star may have little effect on the orbit because the planet has only a small portion of the angular momentum budget, spin synchronization of a star by a planet tends to shrink the orbit, except in the case where the star spins faster than the mean motion. For mature systems comprised of a hot Jupiter and a solar-type star, this is never the case. In fact such systems tend to be unstable to orbital decay because there is not enough angular momentum in the orbit to spin up the star (Rasio et al. 1996), and this is exacerbated if the star is losing angular momentum via a stellar wind. On the other hand, if the star has a convective envelope which is sufficiently decoupled from the core, the synchronization process may only involve this layer and can be achieved before the planet fills its Roche lobe.

For a planet-star system, the timescale for circularization of the orbit, $\tau_{\text {circ }}$, is given by contributions from tides raised on the planet by the star and tides raised on the star by the planet, and is such that

$$
\tau_{\text {circ }}^{-1}=\tau_{e p}^{-1}+\tau_{e *}^{-1}
$$

where

$$
\tau_{e p}=0.16\left(\frac{k_{p}}{0.3}\right)^{-1}\left(\frac{Q_{p}}{10^{5}}\right)\left(\frac{M_{p}}{M_{J}}\right)\left(\frac{M_{*}}{M_{\odot}}\right)^{-1}\left(\frac{R_{p}}{R_{J}}\right)^{-5}\left(\frac{a}{0.04 \mathrm{AU}}\right)^{5} \mathrm{Gyr},
$$

is the contribution to orbital circularization from tides raised in the planet and

$$
\tau_{e *}=\left(\frac{Q_{*}}{Q_{p}}\right)\left(\frac{k_{p}}{k_{*}}\right)\left(\frac{M_{*}}{M_{p}}\right)^{2}\left(\frac{R_{p}}{R_{*}}\right)^{5} \tau_{e p}
$$

is the contribution from tides raised in the star. Here the various symbols have their obvious meaning. For a $1 M_{J}$ planet orbiting a $1 M_{\odot}$ star, $\tau_{e *} \simeq 10\left(Q_{*} / Q_{p}\right)\left(k_{p} / k_{*}\right) \tau_{e p} \gg$ $\tau_{e p}$ for reasonable values of stellar and planetary $Q$-values and Love numbers so that, in this case, it is tides raised in the planet which govern the circularization of the orbit.

In the case where no angular momentum is lost from the system, one can write down timescales for spin-orbit synchronization of the star and planet, $\tau_{\Omega *}$ and $\tau_{\Omega p}$, their spin-orbit alignment, $\tau_{i *}$ and $\tau_{i p}$, and orbit decay or expansion, $\tau_{a}$, all in terms of $\tau_{e p}$ and $\tau_{e *}$ (Hut 1981). These are

$$
\tau_{\Omega_{*}}=\frac{7}{3\left(\alpha_{*}-3\right)} \tau_{e *}, \quad \tau_{\Omega_{p}}=\frac{7}{3\left(\alpha_{p}-3\right)} \tau_{e p},
$$




$$
\tau_{i_{*}}=\frac{7}{\left(\alpha_{*}+1\right)} \tau_{e *}, \quad \tau_{i_{p}}=\frac{7}{\left(\alpha_{p}+1\right)} \tau_{e p}, \quad \text { and } \quad \tau_{a}^{-1} \simeq \tau_{\Omega_{*}}^{-1},
$$

where $\alpha_{*}$ is the ratio of the orbital angular momentum to the body's spin angular momentum at equilibrium, that is, when the star is in the spin-synchronous state, and $\alpha_{p}$ is the planet analogue. ${ }^{8}$ For a Jupiter-mass planet orbiting at a distance of 0.04 $\mathrm{AU}$ from a solar-mass star which has a rotation period of 20 days, $\alpha_{p} \simeq 27000$ so that $\tau_{\Omega_{p}} \ll \tau_{\text {circ }}$ and $\tau_{i_{p}} \ll \tau_{c i r c}$, while there is no stable synchronous state for the star. In fact there is no such state for the star for systems with orbital periods less than 12.7 days for the case where the whole star is spun up. In general, there is no stable state when $\alpha_{*}<3$ (Councelman 1973).

In the case that the star's convective envelope is decoupled from its core, $\alpha_{*}$ should be scaled by $\left(M_{*} / M_{\text {env }}\right)\left(R_{*} / R_{\text {env }}\right)^{2}$, where $M_{\text {env }}$ and $R_{\text {env }}$ are the mass and depth of the convective zone, in which case there will be stable solutions for systems with smaller orbital periods. It has been suggested by Winn et al. (2010) that high stellar obliquities are initially generic to all hot Jupiters, and that the convective envelopes of late-type stars align on timescales shorter than the age of the system while the radiative envelopes of more massive stars are unable to do so.

\section{Conclusion}

Tidal interactions between exoplanets and their host stars are proving to play an extremely important role in our knowledge and understanding of the origin, internal structure and dynamical evolution of short-period planets and their companions. While the eccentricities of most short-period exoplanets are more or less consistent with expectations based on our knowledge of the $Q$-values of the Solar System planets, we still have a long way to go before we fully understand tidal dissipation in the wide variety of circumstances exoplanets present us. In the meantime, the simple equilibrium tide model serves us well in our quest to understand the complex dynamics of single and multiplanet systems.

\section{References}

Butler R. P., Vogt S. S., Marcy G. W., et al., 2004, ApJ, 617, 580

Councelman, C. C. 1973, ApJ, 180, 307

Darwin, G. H. 1879, Phil. Trans. Roy. Astr. Soc., 170, 1

Eggleton, P. P., Kiseleva, L. G., \& Hut, P. 1998, ApJ, 499, 853

Goldreich, P. 1963, MNRAS, 126, 257

Goldreich, P., \& Soter, S. 1966, Icarus, 5, 375

Hut, P. 1981, A\&A, 99, 126

Lainey, V., Arlot, J.-E., Karatekin, Ö., \& van Hoolst, T. 2009, Nature, 459, 957

Rasio, F. A., Tout, C. A., Lubow, S. H., \& Livio, M. 1996, ApJ, 470, 1187

Winn, J. N., Fabrycky, D., Albrecht, S., \& Johnson, J. A. 2010, ApJL, 718, L145

\footnotetext{
${ }^{8}$ The quantity $\alpha_{*}$ is determined by insisting on conservation of angular momentum and solving for the semimajor axis corresponding to $\Omega_{*}=n$ and similarly for $\alpha_{p}$. Since the timescales for spinorbit synchronization of the star and the planet are so different, one can ignore the planet's angular momentum when calculating $\alpha_{*}$, and similarly one can ignore the star's angular momentum when solving for $\alpha_{p}$.
} 